\title{
Bevacizumab treatment for newly diagnosed glioblastoma: Systematic review and meta-analysis of clinical trials
}

\author{
PENG FU $^{1^{*}}$, YUN-SONG HE ${ }^{1 *}$, QIN HUANG ${ }^{2 *}$, TAO DING $^{1}$, YONG-CUN CEN $^{1}$, \\ HONG-YANG ZHAO ${ }^{1}$ and XIANG WEI ${ }^{1}$ \\ Departments of ${ }^{1}$ Neurosurgery and ${ }^{2}$ Orthopedics, Union Hospital, Tongji Medical College, \\ Huazhong University of Science and Technology, Wuhan, Hubei 430022, P.R. China
}

Received February 25, 2015; Accepted November 23, 2015

DOI: $10.3892 / \mathrm{mco} .2016 .816$

\begin{abstract}
High-grade glioma is a richly neovascularized brain solid tumor with a poor prognosis. Bevacizumab is a recombinant humanized monoclonal antibody that inhibits vascular endothelial cell proliferation and angiogenesis, which has shown clinical efficacy in recurrent glioblastoma. MEDLINE/PubMed, EMBASE and Web of Science databases were searched for relevant studies that compared bevacizumab plus combined radiotherapy/temozolomide (RT/TMZ) with RT/TMZ alone in newly diagnosed glioblastoma (GBM). Of all the studies identified, three comparative trials were included in the systematic review. All three enrolling trials, including a total of 1,738 patients, investigated bevacizumab or placebo plus combined RT/TMZ treatment in glioblastoma. The result showed no increased overall survival (OS) (pooled hazard ratio (HR), 1.04; $95 \%$ confidence interval (CI), 0.84-1.29; $\mathrm{P}=0.71)$ but increased progression-free survival (HR, 0.74; 95\% CI, 0.62-0.88; $\mathrm{P}=0.0009)$. However, the two randomized double-blind placebo-control trials exemplified a high rate of adverse events of the bevacizumab compared with the placebo group while discrepant points were noted in term of quality-of-life outcome. Additionally, bevacizumab plus RT/TMZ did not increase the 6-month survival rate [odd ratios (ORs), 0.65; 95\% CI, 0.37-1.13; $\mathrm{P}=0.13$ ). Overall, addition of bevacizumab to radiotherapy-temozolomide treatment may be an effective therapy strategy for improving progression-free survival. OS and the 6-month survival rate was not prolonged and there was questionable efficacy of bevacizumab on the quality-of-life of glioblastoma patients, thus further clinical trials should be performed.
\end{abstract}

Correspondence to: $\mathrm{Dr}$ Peng Fu or Professor Xiang Wei, Department of Neurosurgery, Union Hospital, Tongji Medical College, Huazhong University of Science and Technology, Wuhan, Hubei 430022, P.R. China

E-mail: union_fupeng@163.com

E-mail: xiangwei20@hotmail.com

${ }^{*}$ Contributed equally

Key words: glioblastoma, bevacizumab, radiotherapy, overall survival, temozolomide, progression-free survival

\section{Introduction}

Glioblastoma (GBM) is the most common and most devastating primary intracranial tumor (1). Despite the recommended standard treatment of a combined strategy of maximum safe resection, radiotherapy (RT) with concurrent and adjuvant temozolomide (TMZ) for adult patients with GBM, the typical survival is only 12-15 months from the time of diagnosis (1-3). Thus, there is a requirement for developing a novel therapeutic strategy.

By contrast, malignant gliomas were highly neovascularizated tumors with a distinct phenomenon, angiogenesis and vasculogenesis (4). Initial studies showed that vascular endothelial growth factor A (VEGF-A) was overexpressed in malignant gliomas and had a pivotal role in the processes of angiogenesis and vasculogenesis $(4,5)$. Therefore, VEGF became a fundamental target of antiangiogenic therapy (6). Bevacizumab (BV), also known as avastin, was a humanized monoclonal antibody that directly targets the VEGF-A ligand and can inhibit vascular endothelial cells proliferation and angiogenesis $(7,8)$. Additionally, preclinical evidence indicated that antiangiogenic therapies could result in temporary vascular normalization, potentially improving the efficacy of RT and chemotherapy (6,7). In May 2009, the Food and Drug Administration approved bevacizumab for the first-line treatment of recurrent GBM patients (9). For recurrent GBM patients, the addition of bevacizumab partly improved progression-free survival (PFS) and maintenance of baseline quality of life and performance status $(3,10,11)$, but no improved overall survival (OS) benefit or high-rate adverse events could be observed in these studies. Although Khasraw et al (12) reviewed the benefits and side effects associated with the treatment of antiangiogenic agents for high-grade gliomas, there is no review focusing on bevacizumab for primary glioblastoma. Therefore, a meta-analysis was conducted based on available eligible trials to evaluate the efficacy of bevacizumab combination with RT/TMZ in newly diagnosed glioblastoma patients in terms of OS, progression-free survival and the adverse events rate.

\section{Patients and methods}

Data search and study selection. The identification of literature was performed in two steps and the same principle was 
used to search each database. Two independent review authors searched MEDLINE/PubMed, EMBASE and Web of Science databases for studies published between January 1, 2000 and August 4, 2014. The following keywords were used for systemic searches: Bevacizumab, avastin, maligant glioma, glioblastoma, TMZ, RT and clinical trial. Associated studies and their reference lists were also reviewed individually. In the subsequent step, the following text terms were used: Progression-free survival, OS and 6-month survival rate. All the included studies were searched for the eligible trials by one review author and double-checked by a second review author.

The included trials were only limited to English language and human trials. The included trials were not limited to randomized trials or glioblastoma [World Health Organization (WHO) grade IV], and anaplastic glioma (WHO grade III) was also included. Duplications of the same trials were excluded. Literature that was not published as full studies, such as reference abstracts and letters to editors, were excluded.

Data extraction and quality assessment. Two authors (Peng Fu and Wei Xiang) independently extracted the data from the trials and compared the following results to avoid the bias in this process; all disagreements were resolved by discussion. The following data were obtained from each trial to compare the bevacizumb-based therapies with primary therapy arms: The first author's name, the corresponding author, the year of publication, the number of enrolled patients, the duration of follow-up, the therapy region, hazard ratios (HRs) for OS and PFS, odd ratios (ORs) for 6-month survival rate (SMSR) and adverse events.

Outcome measures and statistical analysis. Types of outcome measures included: i) OS defined as the time interval from the date of diagnosis or treatment to the date of fatality or last follow-up; ii) PFS defined as the time interval from the date of diagnosis or treatment to date of confirmed disease progression; and iii) adverse effects classified according to the WHO criteria, and instances consist of anaemia, neutropenia, leucopaenia, thrombocytopaenia, nausea and vomiting, hypertension, fatigue, thromboembolic disease, hemorrhage, visceral perforation and others.

HRs were used to analyze OS and PFS. If HRs were not reported in the original publications, the HR values and their 95\% confidence intervals (CIs) in each trial would be calculated using the methods described by Tierney et al (13). The estimated HR template that outputs results based on the data of the Kaplan-Meier's survival distributions in search and control groups was used from primary trials. Heterogeneity of the included trials was calculated using the $\chi^{2}$ and $\mathrm{I}^{2}$ statistic, with $\mathrm{P}<0.1$ or $\mathrm{I}^{2}>50 \%$ considered to indicate a statistically significant difference (14). Based on the heterogeneity, the inverse-variance approach was implemented using either fixed- or random-effect models.

\section{Results}

Overview and characteristics of the studies. The search strategy is shown in Table I. All the relevant studies were identified by the initial literature search. Following screening using the keywords, relevant terms and full text information,
Table I. Search strategy in the review.

\begin{tabular}{|c|c|c|}
\hline Database $^{\mathrm{a}}$ & Step & Strategy \\
\hline $\begin{array}{l}\text { MEDLINE/PubMed, } \\
\text { EMBASE and } \\
\text { Web of Science }\end{array}$ & $\begin{array}{r}1 \\
2 \\
3 \\
4 \\
5 \\
6 \\
7 \\
8 \\
9 \\
10 \\
11 \\
12 \\
13 \\
14 \\
15 \\
16 \\
17 \\
18 \\
19 \\
20 \\
21 \\
22\end{array}$ & $\begin{array}{l}\text { Explode 'clinical-trial'/all subheadings } \\
\text { Clinical near trial } \\
\text { Single } \\
\text { Double } \\
\text { Single, double or triple near blind or mas } \\
\text { Random } \\
\text { Control } \\
\text { \#1 or } 2 \text { or } 3 \text { or } 4 \text { or } 5 \text { or } 6 \text { or } 7 \\
\text { EC = 'HUMAN' } \\
\text { \# and } 9 \\
\text { Explode 'brain-tumor'/all subheadings } \\
\text { Malignant glioma } \\
\text { Glioblastoma multiform } \\
\text { Astrocytoma or anaplastic astrocytoma } \\
\text { Avastin } \\
\text { Brain tumor } \\
\text { \#11 or } 12 \text { or } 13 \text { or } 14 \text { or } 15 \text { or } 16 \\
\text { Bevacizumab } \\
\text { Temozolomide } \\
\text { Radiotherapy } \\
\# 18 \text { or } 19 \text { or } 20 \\
\text { \#10 and } 17 \text { and } 21\end{array}$ \\
\hline
\end{tabular}

${ }^{a}$ Original search strategy has been adapted from the Ovid to Silver Platter version. The same principle was suitable for each database search (between January 1, 2000 and August 4, 2014).

studies that were duplicated publications of the same trials and not published as full studies and non-clinical trials were all excluded. The included studies were all only limited to the English language and human trials. One study was excluded (15) as it was an open-label, single-arm clinical trial and lacked comparable data from the control group. Eventually, three studies met the inclusion criteria and were included in the final meta-analysis.

The main characteristics of the included studies are presented in Table II. These three studies represented 1,738 patients, with 848 assigned to the study group to receive bevacizumab plus RT/TMZ and 890 derived for comparison. One belonged to the phase II study and used results derived from a comparable control cohort of patients treated at the University of California (California, LA, USA) and Kaiser Permanente Los Angeles (KPLA) and from the European Organization for Research and Treatment of Cancer-National Cancer Institute of Canada cohort as a comparison (16). The other studies identified randomized, double-blind, placebo-controlled trials $(17,18)$. In both trials, patients were randomly assigned to receive bevacizumab and placebo, and therefore, data from these would provide a more efficacious outcome of the bevacizumab combination with RT/TMZ in GBM patients. All the patients were continued on each treatment until disease progression or unacceptable toxic effects developed.

OS and 6-month survival are not prolonged. OS data were reported in all the studies (16-18). Patients in the bevacizumab 
A

Overall survival

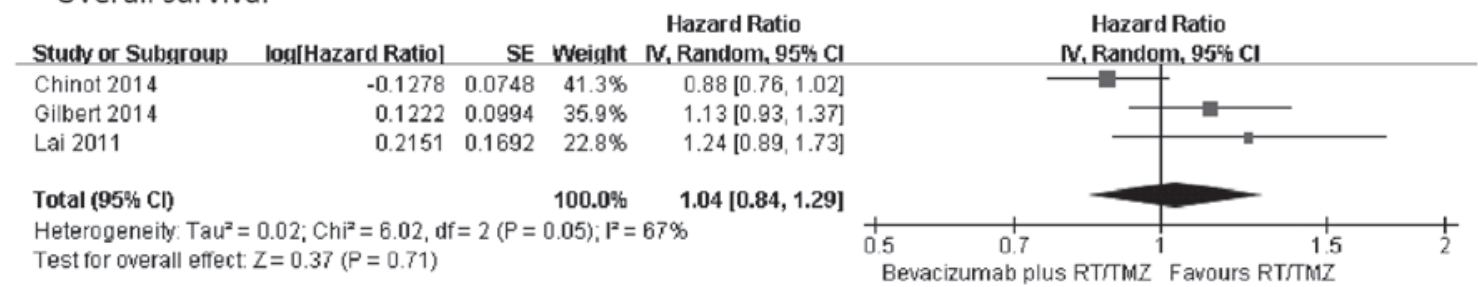

B Progression-free survival Studv or Subgroup logiHazard Ratiol SE Weight $\mathrm{N}$, Random, 95\% Cl

$\begin{array}{lrrrr}\text { Chinot } 2014 & -0.4463 & 0.0773 & 42.0 \% & 0.64[0.55,0.74]\end{array}$
Gilbert $2014 \quad-0.2357 \quad 0.0917 \quad 37.5 \% \quad 0.79[0.66,0.95]$ Lai $2011 \quad-0.1393 \quad 0.1647 \quad 20.5 \% \quad 0.87[0.63,1.20]$

Total (95\% Cl)

$100.0 \% \quad 0.74[0.62,0.88]$

Heterogeneity: Tau $^{2}=0.01 ; \mathrm{Chi}^{2}=4.66, \mathrm{df}=2(P=0.10) ; \mathrm{I}^{2}=57 \%$

Test for overall effect: $Z=3.31(P=0.0009)$

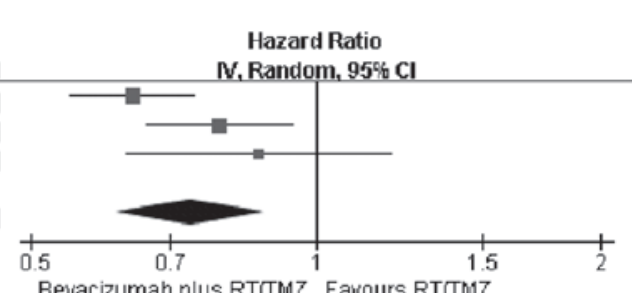

Figure 1. Forest plot of comparison. Bevacizumab plus radiotherapy/temozolomide (RT/TMZ) versus RT/TMZ outcome. (A) Overall survival; (B) progression-free survival.

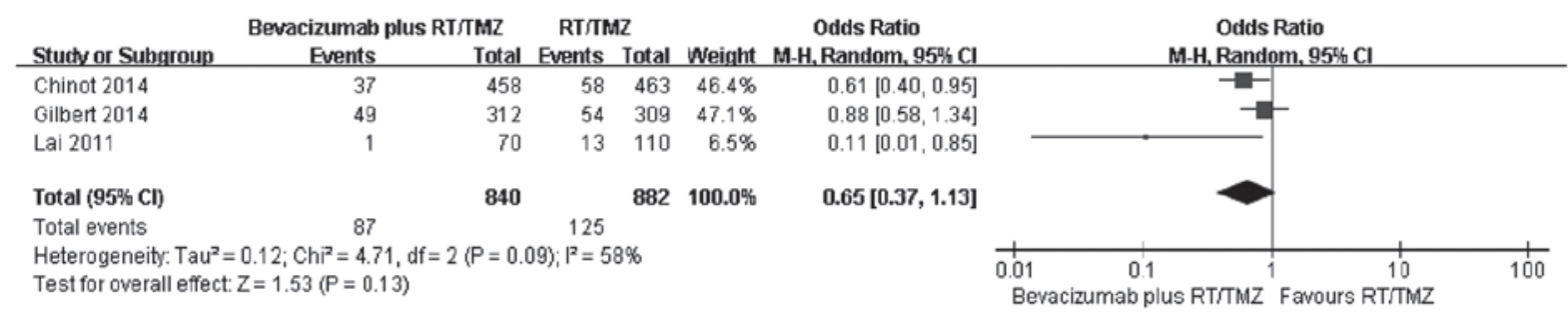

Figure 2. Forest plot of comparison. Bevacizumab plus radiotherapy/temozolomide (RT/TMZ) versus RT/TMZ outcome. Odd ratios for 6-month survival rate.

plus RT/TMZ group had a median OS ranging from 15.7 to 19.6 months, and patients who received primary therapy alone had a median OS ranging from 16.1 to 21.1 months. Only two studies provided adequate data and could be included in the pooled analysis using random-effect meta-analysis, which showed no statistical difference in the median OS between the two groups (HR, 0.99; 95\% CI, 0.77-1.26; heterogeneity $\chi^{2}=4.04, \mathrm{P}=0.04, \mathrm{I}^{2}=75 \%$ ) (Fig. 1A). In order to elucidate the early benefit, the 6-month survival rate was used to evaluate effectiveness of bevacizumab plus RT/TMZ.

A comparison of the 6-month survival rate was conducted from three studies (Fig. 2). As there was large heterogeneity among the studies subsequent to pooling the data $\left(\chi^{2}=4.71\right.$, $\mathrm{df}=2, \mathrm{P}=0.09 ; \mathrm{I}^{2}=58 \%$ ), a random-effects model was used for the meta-analysis of 6-month survival. The combined OR did not reveal a significantly higher survival among patients in the bevacizumab plus RT/TMZ group compared to those in the primary therapy alone group. The ORs in the three studies ranged from 0.11 to 0.88 , with a pooled estimate of $0.65(\mathrm{P}=0.13 ; 95 \% \mathrm{CI}, 0.37-1.13)$. The number of eligible trials providing variation-related estimates was small. Thus, the results of subgroup analyses of OS could not be reported.

Improvement of PFS. Three trials all presented PFS data and reported PFS as the primary end-point (16-18). The median PFS ranged from 10.6-13.6 months in the bevacizumab plus RT/TMZ group and 6.2-7.6 months in the control group. By using a random-effect model, a meta-analysis for PFS from two studies indicated a $29 \%$ reduction in the risk of progression or fatality in patients who received bevacizumab plus RT/TMZ (HR, 0.71; 95\% CI, 0.58-0.87; $\mathrm{P}=0.001$ ). There was statistical heterogeneity between individual trials (heterogeneity $\chi^{2}=3.08$, $\mathrm{P}=0.08, \mathrm{I}^{2}=68 \%$ ) (Fig. 1B).

Bevacizumab-associated adverse events. The serious adverse events (grade $\geq 3$ ) associated with bevacizumab described in the studies included in the meta-analysis are shown in Table III. The included studies showed different conclusions in GBM patients for combination therapy of bevacizumab and RT/TMZ. Although there were certain common nonhematological toxicities (grades III and IV), including fatigue, venous thrombosis, hypertension and proteinuria, the frequency of which was 20,19, 11 and $11 \%$, respectively, in one study (16), hematological toxicities were similar in the patients treated with bevacizumab plus RT/TMZ compared to those in the KPLA and EORTC-NCIC control group and the addition of bevacizumab to $\mathrm{RT} / \mathrm{TMZ}$ did not potentiate hematological toxicity. Notably, the most common nonhematological toxicity, fatigue, exhibited a similar level between the study group and the EORTC-NCIC trial. The other two studies generally agreed with the higher rate of serious adverse events associated with bevacizumab compared with placebo, and increased prevalence and symptoms were more frequent over therapy time in the former group $(17,18)$. Serious adverse events that were observed more frequently in the bevacizumab group included hypertension (11.3 vs. $2.2 \%$ ), proteinuria (5.4 vs. $0 \%$ ), thrombocytopenia (15 vs. $9.8 \%$ ), bleeding (3.3 vs. $1.8 \%$ ) and complications of wound healing (3.3\% vs. $1.6 \%)$ in one 


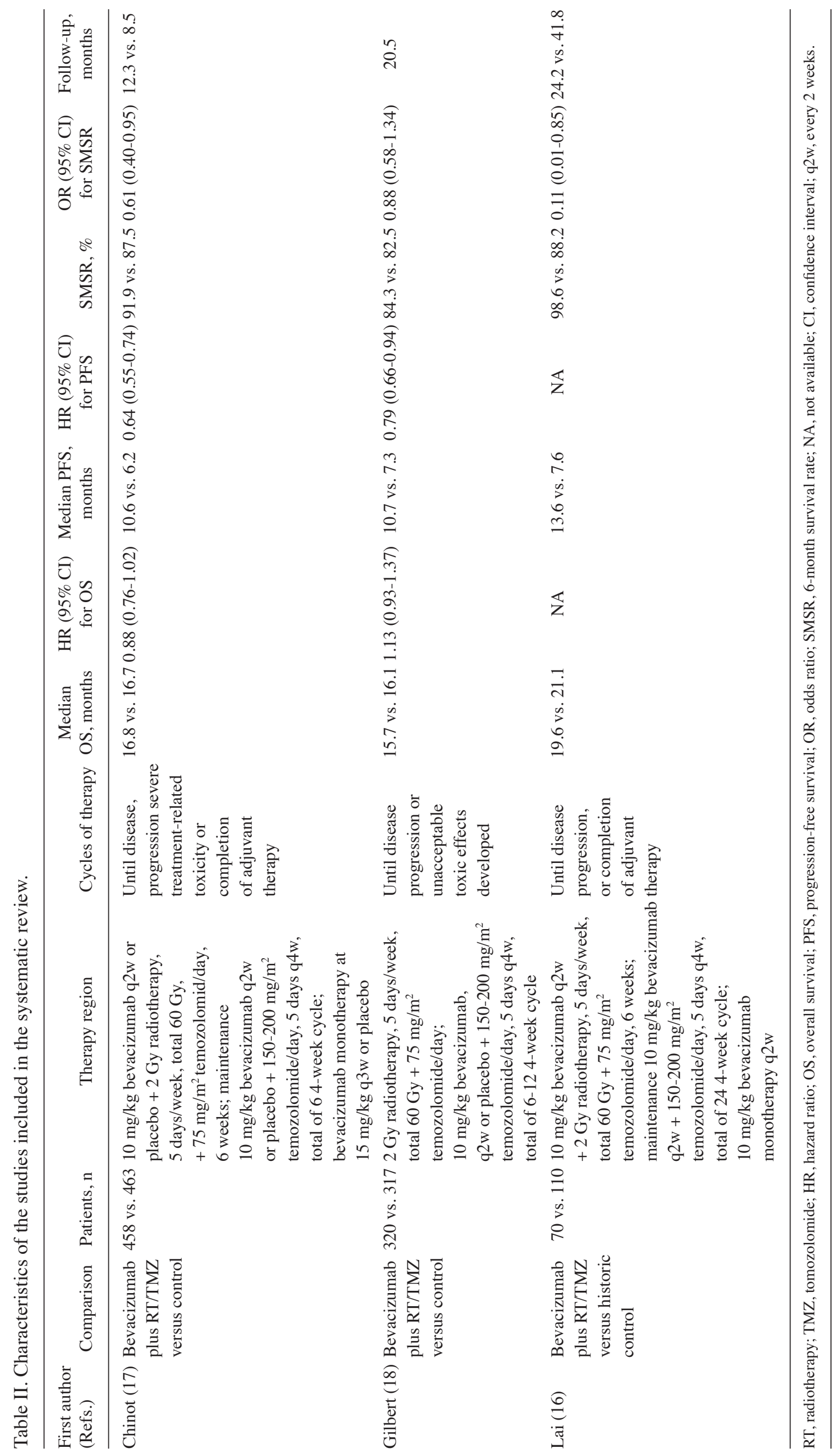


study, while there were more common serious neutropenia (7.3 vs. $3.7 \%$ ) and serious thrombocytopenia (10.2 vs. $7.7 \%$ ) during chemoradiotherapy with bevacizumab or placebo and hypertension $(4.2$ vs. $0.9 \%)$ and serious neutropenia (10.0 vs. $5.1 \%$ ) during the maintenance phase in the bevacizumab group compared to the placebo group in the other. The adverse events often associated with bevacizumab treatment mainly included hypertension, proteinuria, thromboembolic events, complications associated with wound healing, gastrointestinal perforation, bleeding and hematological toxicity.

\section{Discussion}

The present meta-analysis included two randomized double-blind placebo-control trials (RCTs) plus one open-label single-arm clinical trial. The results showed significantly improved PFS in primary glioblastoma patients treated with bevacizumab plus RT/TMZ versus those only treated with radiotherapy with concurrent and adjuvant chemotherapeutic choices. However, there was no significant different in OS. The pooled result from two RCTs for PFS was 0.71 (95\% CI, 0.58-0.87; $\mathrm{P}=0.0010)$, whereas the pooled result from two RCTs for OS and odd ratios (ORs) for SMSR from three studies were 0.99 (95\% CI, 0.77-1.26; $\mathrm{P}=0.93$ ) and 0.65 (95\% CI, 0.37-1.13; $\mathrm{P}=0.13$ ), respectively. Thus, despite a combination treatment of bevacizumab and RT/TMZ that significantly improved the PFS in patients with GBM, this treatment could not prolong the median OS and SMSR. A possible explanation may be that tumors have become resistant to bevacizumab or that the vasculature may regrow rapidly following the termination of bevacizumab treatment. In a recent meta-analysis, Khasraw et al (12) evaluated the efficacy and toxicity of antiangiogenic therapy in patients with high-grade glioma and showed that bevacizumab was the antiangiogenic therapy more likely to yield favorable results. This analysis had similar pooled HR for PFS for three bevacizumab studies with 1,712 participants, which indicated significant improvement at 0.66 (95\% CI, 0.59-0.74; $\mathrm{P}<0.00001$ ). This finding was also not significant for OS (HR, 0.92; 95\% CI, 0.83-1.02; $\mathrm{P}=0.12$ ). The study by Takano et al (19) reported the outcomes in Japanese patients, and Japan was the only country to approve the use of bevacizumab in combination with RT and TMZ chemotherapy for newly diagnosed glioblastoma, and PFS and OS (12.2 and 29.2 months at median, respectively) was shown to be longer for those treated with bevacizumab than for those not treated with the drug.

In the present analysis, three trials were reviewed and the heterogeneity of OS $\left(\chi^{2}=4.04, \mathrm{P}=0.04, \mathrm{I}^{2}=75 \%\right)$, ORs for SMSR $\left(\chi^{2}=4.71, \mathrm{P}=0.09, \mathrm{I}^{2}=58 \%\right)$ and PFS $\left(\chi^{2}=3.08, \mathrm{P}=0.08, \mathrm{I}^{2}=68 \%\right)$ were calculated. As $\mathrm{P}<0.1$ or $\mathrm{I}^{2}>50 \%$ was considered to indicate a statistically significant difference (14), the resources of the difference were considered, including the number of trials, study design, treatment regimen and evaluation criteria of outcomes. As an example using therapy, the strategy with becacizumab was concurrently added to RT/TMZ in early stage and subsequently adjuvant with TMZ in two studies, whereas treatment with bevacizumab began during week 4 of $\mathrm{RT}$ and was continued for cycles of maintenance chemotherapy in the trial by Gilbert et al (18). However, despite these differences, the present results strongly suggest that the addition of 
bevacizumab could confer a PFS benefit in patients with newly diagnosed GBM, with evidence of not prolonging OS and the 6-month survival rate.

Bevacizumab as an antiangiogenic agent has been approved for recurrent glioblastoma due to high response rates. A recent comprehensive review of toxicities experienced among 3 phase II bevacizumab trials for recurrent malignant gliomas at the National Cancer Institute showed bevacizumab monotherapy is well-tolerated, however, toxicity increases with combination therapy (20). Although the three included studies reported evidence of toxicity and serious adverse events associated with bevacizumab, Lai et al (16) concluded toxicity attributable to RT/TMZ was similar and additional toxicities were consistent with those reported in other bevacizumab trials and the discrepancy of quality-of-life findings from bevacizumab treatment was founded between Chinot et al (17) and Gilbert et al (18). Chinot et al (17), whose trial was sponsored by a pharmaceutical company, showed maintenance of quality-of-life outcomes, whereas Gilbert et al (18), whose trial was sponsored by the National Cancer Institute, showed decreased quality of life. The reason of the discrepancy may be associated with the different evaluation criteria of quality-of-life measures, distinguishing limited health-related quality-of-life analyses based on time to either deterioration or tumor progression from above, as well as additional tests of symptom burden, interference and neurocognitive function. Therefore, it is essential to acknowledge the spectrum of bevacizumab toxicities and predisposing risk factors to weight the risks and benefits of bevacizumab.

Several factors and limitations should be considered when interpreting the present results. For example, a major strength of the study is that there were only two available data sets from RCTs, which were well performed and of high quality; published in the New England Journal of Medicine. All the included studies have an enlarged sample size of 1,738 , and a statistical power was enhanced to provide reliable effect estimates. Although the present meta-analysis represents a general summary of the efficacy of bevacizumab combined with RT/TMZ on patients with GBM, it also worth emphasizing the potential limitations. Firstly, the data were extracted from publications rather than individual patient estimations, so the treatment benefits may not be defined clearly. Secondly, the cost-effectiveness of bevacizumab treatment was not taken into consideration as it was a type of biological preparation, the cost of which remains relatively high, objectively defining the cost-effectiveness as not easy. Thirdly, the small number of studies included, heterogeneity on the basis of a statistical design and therapy regimen of three clinical studies may lead to somewhat bias, which makes it a challenge to draw a significant conclusion, and additional trials are warranted to obtain more associated data.

In conclusion, the results of the present systematic review and meta-analysis show that the therapy of bevacizumab combined with RT/TMZ offers a statistically significant improvement in PFS in patients with newly diagnosed GBM, but does not benefit OS and SMSR. Bevacizumab combined with RT/TMZ should be suggested for first-line treatment in GBM patients as early as possible to experience the early-stage benefits, but ongoing well-designed clinical trials are also required until statistically significant OS and adverse events differences are documented.

\section{References}

1. Wen PY and Kesari S: Malignant gliomas in adults. N Engl J Med 359: 492-507, 2008.

2. Stupp R, Hegi ME, Mason WP, van den Bent MJ, Taphoorn MJ, Janzer RC, Ludwin SK, Allgeier A, Fisher B, Belanger K, et al; European Organisation for Research and Treatment of Cancer Brain Tumour and Radiation Oncology Groups; National Cancer Institute of Canada Clinical Trials Group: Effects of radiotherapy with concomitant and adjuvant temozolomide versus radiotherapy alone on survival in glioblastoma in a randomised phase III study: 5-year analysis of the EORTC-NCIC trial. Lancet Oncol 10: 459-466, 2009.

3. Stupp R, Mason WP, van den Bent MJ, Weller M, Fisher $\mathrm{B}$, Taphoorn MJ, Belanger $\mathrm{K}$, Brandes AA, Marosi C, Bogdahn U, et al; European Organisation for Research and Treatment of Cancer Brain Tumor and Radiotherapy Groups; National Cancer Institute of Canada Clinical Trials Group: Radiotherapy plus concomitant and adjuvant temozolomide for glioblastoma. N Engl J Med 352: 987-996, 2005.

4. Jain RK, di Tomaso E, Duda DG, Loeffler JS, Sorensen AG and Batchelor TT: Angiogenesis in brain tumours. Nat Rev Neurosci 8: 610-622, 2007.

5. Burrell K, Singh S, Jalali S, Hill RP and Zadeh G: VEGF regulates region-specific localization of perivascular bone marrow-derived cells in glioblastoma. Cancer Res 74: 3727-3739, 2014.

6. Jain RK: Normalization of tumor vasculature: An emerging concept in antiangiogenic therapy. Science 307: 58-62, 2005.

7. Ferrara N, Hillan KJ and Novotny W: Bevacizumab (Avastin), a humanized anti-VEGF monoclonal antibody for cancer therapy. Biochem Biophys Res Commun 333: 328-335, 2005.

8. Robles Irizarry L, Hambardzumyan D, Nakano I, Gladson CL and Ahluwalia MS: Therapeutic targeting of VEGF in the treatment of glioblastoma. Expert Opin Ther Targets 16: 973-984, 2012.

9. Johnson DR, Leeper HE and Uhm JH: Glioblastoma survival in the United States improved after Food and Drug Administration approval of bevacizumab: A population-based analysis. Cancer 119: 3489-3495, 2013.

10. Ahluwalia MS and Gladson CL: Progress on antiangiogenic therapy for patients with malignant glioma. J Oncol 2010: 689018, 2010.

11. Narayana A, Kelly P, Golfinos J, Parker E, Johnson G, Knopp E, Zagzag D, Fischer I, Raza S, Medabalmi P, et al: Antiangiogenic therapy using bevacizumab in recurrent high-grade glioma: Impact on local control and patient survival. J Neurosurg 110: 173-180, 2009.

12. Khasraw M, Ameratunga MS, Grant R, Wheeler $\mathrm{H}$ and Pavlakis N: Antiangiogenic therapy for high-grade glioma. Cochrane Database Syst Rev 9: CD008218, 2014.

13. Tierney JF, Stewart LA, Ghersi D, Burdett S and Sydes MR: Practical methods for incorporating summary time-to-event data into meta-analysis. Trials 8: 16, 2007.

14. Higgins JP, Thompson SG, Deeks JJ and Altman DG: Measuring inconsistency in meta-analyses. BMJ 327: 557-560, 2003.

15. Narayana A, Gruber D, Kunnakkat S, Golfinos JG, Parker E, Raza S, Zagzag D, Eagan P and Gruber ML: A clinical trial of bevacizumab, temozolomide, and radiation for newly diagnosed glioblastoma. J Neurosurg 116: 341-345, 2012.

16. Lai A, Tran A, Nghiemphu PL, Pope WB, Solis OE, Selch M, Filka E, Yong WH, Mischel PS, Liau LM, et al: Phase II study of bevacizumab plus temozolomide during and after radiation therapy for patients with newly diagnosed glioblastoma multiforme. J Clin Oncol 29: 142-148, 2011.

17. Chinot OL, Wick W, Mason W, Henriksson R, Saran F, Nishikawa R, Carpentier AF, Hoang-Xuan K, Kavan P, Cernea D, et al: Bevacizumab plus radiotherapy-temozolomide for newly diagnosed glioblastoma. N Engl J Med 370: 709-722, 2014.

18. Gilbert MR, Dignam JJ, Armstrong TS, Wefel JS, Blumenthal DT, Vogelbaum MA, Colman H, Chakravarti A, Pugh S, Won M, et al: A randomized trial of bevacizumab for newly diagnosed glioblastoma. N Engl J Med 370: 699-708, 2014.

19. Takano S, Ishikawa E, Nakai K, Matsuda M, Masumoto T, Yamamoto T and Matsumura A: Bevacizumab in Japanese patients with malignant glioma: From basic research to clinical trial. Onco Targets Ther 7: 1551-1562, 2014.

20. Odia Y, Shih JH, Kreisl TN and Fine HA: Bevacizumab-related toxicities in the National Cancer Institute malignant glioma trial cohort. J Neurooncol 120: 431-440, 2014. 